\title{
Matrix Integration and Combinatorics of Modular Groups
}

\author{
C. Itzykson and J.-B. Zuber \\ Service de Physique Théorique de Saclay ${ }^{\star}$, F-91191 Gif-Sur-Yvette Cedex, France
}

Received January 24, 1990

\begin{abstract}
Integration over Gaussian matrix ensembles was used to obtain the virtual Euler characteristics of mapping class groups. We present some simplifications in the combinatorial part of the calculation.
\end{abstract}

\section{Introduction}

In a beautiful work, Harer and Zagier [1] obtained the (virtual) Euler characteristics of the mapping class (or modular) group of punctured orientable compact Riemann surfaces of genus $g$ in two steps. The first of topological nature is the reduction to a purely combinatorial problem. The second one is the solution of this combinatorial problem using a variety of techniques, including at some stage an integration over a Gaussian ensemble of random matrices. This point was further elaborated by Penner [2] who used a dual version of this integration. Penner made contact with physicists' work initiated by 't Hooft [3] and further developed in [4-7] on the topological interpretation of the large $N$ expansion of (field theoretic) models. In this note we shall not attempt to describe the topological background, referring the reader to the expositions of the previously mentioned authors [1,2] as well as the review by Ivanov [8]. Rather we would like to show that the combinatorial calculations in both references [1] and [2] can be substantially reduced if one appeals to fairly standard tools. We shall present three elementary calculations. The first is purely group theoretical, and relies on the Frobenius duality formula between the linear and symmetric groups. In essence, it had already been sketched in the last appendix of [6] and was based on a suggestion of J.-M. Drouffe. Parenthetically, we obtain a strikingly simple expression for the average of an irreducible polynomial character over the Gaussian Hermitian ensemble [formula (2.13) below], which awaits presumably adequate generalizations.

A second calculation is even simpler and only requires an elementary knowledge of the harmonic oscillator. It is candidate for generalizations based on an interpretation in terms of free fermions, too.

\footnotetext{
^ Laboratoire de l'Institut de Recherche Fondamentale du Commissariat à l'Energie Atomique
} 
For completeness, we also review the combinatorial part of Penner's work showing that there is in fact no real need to introduce orthogonal polynomials [6] to obtain the required expressions in a fairly straightforward way.

\section{Group-Theoretic Method}

We are interested in averages over a Gaussian ensemble of hermitian $N \times N$ matrices $M$ of monomials of degree $2 n$,

$$
\begin{gathered}
\left\langle t_{\underline{v}}\right\rangle=\frac{\int d M t_{\underline{v}}(M) e^{-S}}{\int d M e^{-S}}, \\
S=\frac{1}{2} \operatorname{tr} M^{2}, \\
d M=\prod_{1 \leqq i \leqq N} d M_{i i} \prod_{1 \leqq i<j \leqq N} d\left(\operatorname{Re} M_{i j}\right) d\left(\operatorname{Im} M_{i j}\right), \\
t_{\underline{v}}(M)=(\operatorname{tr} M)^{v_{1}} \ldots\left(\operatorname{tr} M^{p}\right)^{v_{p}} \ldots,
\end{gathered}
$$

where $\sum j v_{j}=2 n$, and the multi-index $\underline{v}$ denotes the partition of $2 n: \underline{y}=\left[1^{v_{1}} \ldots\right.$ $\left.(2 \mathrm{n})^{v_{2 n}}\right]$.

The symmetric group $S_{2 n}$ of permutation of $2 n$ objects will play a central role in our discussion. Its classes are in one-to-one correspondence with partitions $\underline{v}=\left[1^{v_{1}} \ldots(2 n)^{v_{2 n}}\right]$ which allows an abusive identification so that we sometimes use the same notation. To refer to a class of an element $\sigma$ in $S_{2 n}$ we write $[\sigma]$ instead of the heavier notation $\underline{v}(\sigma)$. The number of elements in class $\underline{v}$ reads

$$
|\underline{v}|=\frac{(2 n) !}{\prod_{k} k^{v_{k}} v_{k} !} .
$$

The irreducible characters $\chi^{Y}$ of $S_{2 n}$ are labelled by Young tableaux $Y$.

The "propagator" which follows from (2.2) is simply

$$
\left\langle M_{i j} M_{k l}\right\rangle=\delta_{i l} \delta_{j k}
$$

and may be represented as a double line with opposite orientations (Fig. 1). The quantity $\left\langle t_{y}\right\rangle$ is a sum of products of connected contributions (labelled by the index $a$ ) arising from all possible Wick contractions between the "vertices" $\left(\operatorname{tr} M^{m}\right)^{v_{m}}$. These $\sum V_{a}=V=\sum v_{j}$ vertices and $\sum L_{a}=L=\frac{1}{2} \sum j v_{j}=n$ lines thus build up a set of connected orientable surfaces with $F=\sum F_{a}$ faces (each connected component has a genus $g_{a}$ such that $2-2 g_{a}=V_{a}-L_{a}+F_{a}$ ), which contributes $N^{F}$ to $\left\langle t_{v}\right\rangle$. An alternative procedure to sum all these contributions consists in labelling each double line incident on a vertex by an integer running between 1 and $2 n$. The connectivity of the lines at the vertices then defines a fixed permutation $\sigma$ :
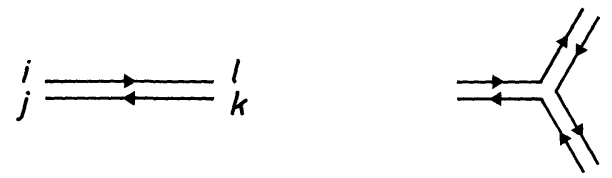

Fig. 1. The double line representation of the propagator and of the vertex $\operatorname{tr} M^{3}$ 


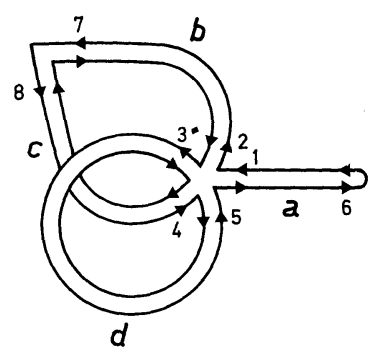

Fig. 2. A typical contribution to $\left\langle\operatorname{tr} M^{5} \operatorname{tr} M^{2} \operatorname{tr} M\right\rangle ; \quad \sigma=(23451687) \in[1,2,5]$ and $\tau=(67583124) \in\left[2^{4}\right]$, hence $\sigma \tau \in[8]$

in the example of Fig. 2, $\sigma$ maps (12345678) onto (23451687); $\sigma$ belongs to the class $[\sigma]=\underline{v}=\left[1^{v_{1}} \ldots 2 n^{v_{2 n}}\right]$. The contractions define another permutation $\tau$, which specifies which pairs of double lines are connected $[\tau=(67583124)$ in Fig. 2$]$. Clearly, $\tau$ is in the class $\left[2^{n}\right]$, and the number $F$ is the number of cycles of $\sigma \tau$. Thus

$$
\left\langle t_{\underline{u}}\right\rangle=\sum_{\substack{\mu \\ \text { partition of } 2 n}} \sum_{\substack{\tau \in\left[2^{n}\right] \\ \sigma \tau \in \underline{\mu}}} N^{\Sigma \mu_{\nu}}=\sum_{\substack{\mu \\ \text { class of } S_{2 n}}} N^{\Sigma \mu_{i}} \sum_{\tau} \delta_{[\tau],\left[2^{n}\right]} \delta_{[\sigma \tau], \underline{\mu}} .
$$

Thanks to the completeness and orthogonality of the real characters $\chi^{Y}$,

$$
\begin{gathered}
\sum_{Y} \chi^{Y}(\sigma) \chi^{Y}(\tau)=\frac{(2 n) !}{|[\sigma]|} \delta_{[\sigma],[\tau]}, \\
\sum_{\tau} \chi^{Y}(\tau) \chi^{Y^{\prime}}(\sigma \tau)=(2 n) ! \delta_{Y Y^{\prime}} \frac{\chi^{Y}(\sigma)}{\chi^{Y}\left(\left[1^{2 n}\right]\right)}
\end{gathered}
$$

this may be rewritten as

$$
\begin{aligned}
\left\langle t_{\underline{v}}\right\rangle & =\sum_{\substack{\underline{\mu} \\
\text { class of } S_{2 n}}} N^{\Sigma \mu_{i}} \sum_{Y, Y^{\prime}} \sum_{\tau} \frac{\left|\left[2^{n}\right]\right||\underline{\mu}|}{((2 n) !)^{2}} \chi^{Y}(\tau) \chi^{Y}\left(\left[2^{n}\right]\right) \chi^{Y^{\prime}}(\sigma \tau) \chi^{Y^{\prime}}(\underline{\mu}), \\
& =\sum_{\substack{\mu \\
\text { class of } S_{2 n}}} N^{\Sigma \mu_{i}} \sum_{Y} \frac{\left|\left[2^{n}\right]\right||\underline{\mu}| \chi^{Y}\left(\left[2^{n}\right]\right) \chi^{Y}(\underline{\mu}) \chi^{Y}(\underline{v})}{(2 n) !} \frac{\chi^{Y}\left(\left[1^{2 n}\right]\right)}{}
\end{aligned}
$$

This formula may be reexpressed by saying that the contribution $\left\langle t_{\underline{v}}\right\rangle_{\underline{\mu}}$ of class $\underline{\mu}$ to $\left\langle t_{\underline{v}}\right\rangle$ is such that

$$
\frac{\left\langle t_{v}\right\rangle_{\underline{\mu}}}{|\underline{\mu}| N^{\Sigma \mu_{i}}}=\frac{\left|\left[2^{n}\right]\right|}{(2 n) !} \sum_{Y} \frac{\chi^{Y}\left(\left[2^{n}\right]\right) \chi^{Y}(\underline{\mu}) \chi^{Y}(\underline{v})}{\chi^{Y}\left(\left[1^{2 n}\right]\right)}
$$

which exhibits a symmetry in the interchange $\mu \leftrightarrow \underline{v}$, reflecting the duality between vertices and faces of the surfaces. Contributions to $\left\langle t_{v}\right\rangle_{\underline{\mu}}$ come from surfaces with $V=\sum v_{j}$ vertices, $L=\frac{1}{2} \sum j v_{j}=\frac{1}{2} \sum j \mu_{j}=n$ lines and $\bar{F}=\sum \mu_{j}$ faces. Duality exchanges $\underline{v}$ and $\mu$.

The irreducible polynomial characters $\mathrm{ch}_{Y}$ of the linear group $G L(N)$ associated with the same set of Young tableaux $Y$ satisfy the Frobenius reciprocity relation [9],

$$
t_{\underline{v}}(M)=\sum_{Y} \operatorname{ch}_{Y}(M) \chi^{Y}(\underline{v}) \text {. }
$$


Since $\operatorname{ch}_{Y}(M)$ is a polynomial in the matrix elements of $M$, Eq. (2.11) can be extended to any matrix, in particular to an hermitian matrix. Taking the average and comparing with (2.9), we find

$$
\left\langle\operatorname{ch}_{Y}\right\rangle=(2 n-1) ! ! \frac{\chi^{Y}\left(\left[2^{n}\right]\right)}{\chi^{Y}\left(\left[1^{2 n}\right]\right)} \sum_{\substack{\mu \\ \text { class of } S_{2 n}}} \chi^{Y}(\underline{\mu}) \frac{N^{\Sigma \mu_{i}}}{\prod \mu_{j} ! j^{\mu_{j}}} .
$$

The last sum is identified as the dimension $\operatorname{ch}_{Y}(1)$ of the representation of the linear group associated with $Y$, using again the Frobenius formula. Thus we find the remarkably simple expression

$$
\left\langle\operatorname{ch}_{Y}\right\rangle=(2 n-1) ! ! \frac{\chi^{Y}\left(\left[2^{n}\right]\right)}{\chi^{Y}\left(\left[1^{2 n}\right]\right)} \operatorname{ch}_{Y}(1),
$$

which admits a trivial generalization in the form

$$
\left\langle\operatorname{ch}_{Y}\left(M M^{\prime}\right)\right\rangle_{M}=(2 n-1) ! ! \frac{\chi^{Y}\left(\left[2^{n}\right]\right)}{\chi^{Y}\left(\left[1^{2 n}\right]\right)} \operatorname{ch}_{Y}\left(M^{\prime}\right),
$$

where the index $M$ on the bracket indicates that the average is taken over $M$.

In conjunction with (2.11), formula (2.13) could be useful in deriving some new results. Here we shall show that it leads to a simple derivation of the combinatorial results of [1]. An intermediate step in the determination of the virtual Euler characteristics $\chi\left(\Gamma^{1}\right)$ of the moduli space of once-punctured orientable compact Riemann surfaces is the calculation of the number $\varepsilon_{g}(n)$ of pairwise identifications of the sides of a $2 n$-gon leading to a surface of genus $g$. This is immediately seen to be equal to the number of contractions between the lines of the $\operatorname{tr} M^{2 n}$ vertex (Fig. 3), hence

$$
\varepsilon_{g}(n)=\sum_{\Sigma \mu_{i}=n+1-2 \mathrm{~g}} \frac{\left\langle t_{[2 \mathrm{n}]}\right\rangle_{\mu}}{N^{\Sigma \mu_{i}}}
$$

computed as above. Combining (2.13) with (2.11) leads to

$$
\sum_{\substack{g \\ 2 g \leqq n}} N^{n+1-2 g} \varepsilon_{g}(n)=\left\langle t_{[2 n]}\right\rangle=(2 n-1) ! ! \sum_{Y} \frac{\chi^{Y}([2 n]) \chi^{Y}\left(\left[2^{n}\right]\right)}{\chi^{Y}\left(\left[1^{2 n}\right]\right)} \operatorname{ch}_{Y}(1) .
$$

The non-vanishing of the characters $\chi^{Y}([2 n])$ restricts the sum over $Y$ to a subclass of Young tableaux, namely those with at most one row of length $q+1 \geqq 1$ and $p \geqq 0$
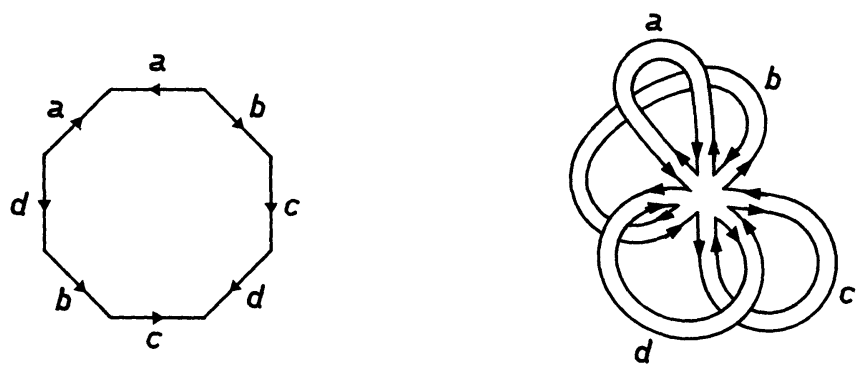

Fig. 3. Equivalence between a pairwise identification of the sides of an octogon and a set of contractions of lines of the vertex $\operatorname{tr} M^{8}$. The graph is dual to the graph of Fig. 2 
rows of length $1, p+q+1=2 n$. All the expressions that appear in (2.16) are quite explicit [6],

$$
\begin{aligned}
& \chi^{Y_{p q}}([2 n])=(-1)^{p}, \\
& \chi^{Y_{p q}}\left(\left[2^{n}\right]\right)=\left\{\begin{array}{cl}
(-1)^{p / 2}\left(\begin{array}{c}
n-1 \\
\frac{p}{2}
\end{array}\right) & \text { if } p \text { is even } \\
(-1)^{\frac{p+1}{2}}\left(\begin{array}{c}
n-1 \\
\frac{p-1}{2}
\end{array}\right) & \text { if } p \text { is odd, }
\end{array}\right. \\
& \frac{\operatorname{ch}_{Y_{p q}}(\mathbf{1})}{\chi^{Y_{p q}\left(\left[1^{2 n}\right]\right)}}=\left(\begin{array}{c}
N+q \\
2 n
\end{array}\right) \text { with } p+q+1=2 n .
\end{aligned}
$$

(The constraint $p+1 \leqq N$ is taken into account by the vanishing of $\frac{1}{(N-p-1) !}$ for $p \geqq N$.) Thus

$$
\begin{aligned}
\frac{\left\langle t_{[2 n]}\right\rangle}{(2 n-1) ! !} & =\sum_{p=0}^{n-1}(-1)^{p}\left(\begin{array}{c}
n-1 \\
p
\end{array}\right)\left[\left(\begin{array}{c}
N+2 n-2 p-1 \\
2 n
\end{array}\right)+\left(\begin{array}{c}
N+2 n-2 p-2 \\
2 n
\end{array}\right)\right] \\
& =\sum_{p=0}^{n-1}(-1)^{p}\left(\begin{array}{c}
n-1 \\
p
\end{array}\right) \oint \frac{d x}{2 i \pi}\left(\frac{(1+x)^{N+2 n-2 p-1}}{x^{N-2 p}}+\frac{(1+x)^{N+2 n-2 p-2}}{x^{N-2 p-1}}\right) \\
& =\oint \frac{d x}{2 i \pi} \frac{(1+x)^{N}(1+2 x)^{n}}{x^{N}} \\
& =\frac{1}{2} \oint \frac{d y}{2 i \pi} \frac{1}{y^{n+2}}\left(\frac{1+y}{1-y}\right)^{N},
\end{aligned}
$$

where $y=1 /(1+2 x)$. This is the form given in [1].

There is an intriguing feature in this last result. A generating function for the characters $\mathrm{ch}_{Y}$ for the Young tableaux $Y_{p q}$ is given by

$$
\operatorname{det}\left(\frac{1+y M}{1-z M}\right)=1+\sum_{p, q \geqq 0}(y+z) y^{p} z^{q} \operatorname{ch}_{Y_{p q}}(M),
$$

which follows from $\operatorname{ch}_{Y_{p, 0}} c h_{Y_{0, q}}=\operatorname{ch}_{Y_{p, q+1}}+\operatorname{ch}_{Y_{p+1}, q}$, where of course $\operatorname{ch}_{Y_{p, q}}$ vanishes if $p \geqq N$. Evaluated for $y=z, M=1$, this is the integrand of the last equation (2.18). Thus this last formula (2.18) involves the same family of Young tableaux, but with the condition that $p+q=n$ instead of $p+q=2 n-1$ in (2.17). The following observation may be in order. Let $p, q$ be non-negative integers with $p+q=n$ fixed, $x_{1}, \ldots, x_{N} N$ indeterminates and $V_{p, q}$ the space of forms

$$
\frac{1}{p !} \sum_{i_{1}, \ldots, i_{p}} P_{i_{1} \ldots i_{p}}\left(x_{1}, \ldots, x_{N}\right) d x_{i_{1}} \ldots d x_{i_{p}},
$$


where the indices $i_{k}$ run from 1 to $N$ and the $P$ 's are homogeneous polynomials of degree $n+1-p=q+1$ so that the total degree in $x$ is $n+1$. The exterior derivative $d$ maps $V_{p-1, q+1}$ into $V_{p, q}$. Then the factor space

$$
E_{p, q}=V_{p, q} \bmod d\left(V_{p-1, q+1}\right)
$$

supports the representation of the linear group corresponding to $Y_{p, q}$ and the direct sum

$$
E=E=\bigoplus_{n} E^{(n)}=\bigoplus_{n} \underset{p+q=n}{\bigoplus_{p, q}} E_{p}
$$

is the graded representation space introduced above. This suggests to look for a simple (cohomological) explanation for the above relations.

\section{Harmonic Oscillators}

In this section, we derive a close expression for the generating function of the $\left\langle t_{[2 n]}\right\rangle$

$$
\begin{aligned}
T(x) & =1+2 \sum_{0}^{\infty} x^{n+1} \frac{\left\langle t_{[2 n]}\right\rangle}{(2 n-1) ! !} \\
& =1+2 \int_{0}^{\infty} d y \sum_{n} \frac{\left\langle t_{[2 n]}\right\rangle y^{2 n+1}}{(2 n) !} e^{-\frac{y^{2}}{2 x}} \\
& =1+2 \int_{0}^{\infty} y d y e^{-\frac{y^{2}}{2 x}}\left\langle\operatorname{tr} e^{y M}\right\rangle
\end{aligned}
$$

since the odd powers do not contribute, $\left\langle t_{[2 n+1]}\right\rangle=0$. The trace may be expressed in terms of the eigenvalues $\lambda_{i}$ of the matrix $M$, and the average is now performed with the measure

$$
D \lambda=\Delta^{2}(\lambda) \prod_{i} d \lambda_{i} e^{-\frac{\lambda_{i}^{2}}{2}},
$$

where $\Delta(\lambda)$ stands for the Vandermonde determinant

$$
\begin{gathered}
\Delta(\lambda)=\prod_{1 \leqq i<j \leqq N}\left(\lambda_{i}-\lambda_{j}\right)=\operatorname{det}\left(\lambda_{i}^{j-1}\right)=\operatorname{det}\left(H_{j-1}\left(\lambda_{i}\right)\right) \\
=\sum_{\text {permutation of } 1, \ldots, N}(-1)^{P} H_{N-1}\left(\lambda_{P_{1}}\right) \ldots H_{0}\left(\lambda_{P_{N}}\right) .
\end{gathered}
$$

The $H_{k}$ denote the orthogonal (Hermite) monic polynomials for the measure $d \lambda e^{-\left(\lambda^{2} / 2\right)} ; H_{k}$ is of degree $k$ and its term of highest degree has coefficient 1 . Thus

$$
\begin{aligned}
\left\langle\operatorname{tr} e^{y M}\right\rangle= & \mathcal{N}^{-1} \int D \lambda \sum_{s} e^{y \lambda_{s}} \\
= & N ! \mathscr{N}^{-1} \int \prod_{i} d \lambda_{\imath} e^{-\frac{\lambda_{i}^{2}}{2}} H_{N-1}\left(\lambda_{1}\right) \ldots H_{0}\left(\lambda_{N}\right) \\
& \times \sum_{P}(-1)^{P} H_{N-1}\left(\lambda_{P_{1}}\right) \ldots H_{0}\left(\lambda_{P_{N}}\right) \sum_{s} e^{y \lambda_{s}}
\end{aligned}
$$


with a normalization factor $\mathcal{N}=(2 \pi)^{N / 2} \prod_{1}^{N} p$ !. When the contribution of the term $e^{y \lambda_{s}}$ is considered, orthogonality forces $P_{j}=j$ for all $j \neq s$, hence $P$ is the identity permutation.

$$
\begin{aligned}
\left\langle\operatorname{tr} e^{y M}\right\rangle & =N ! \mathscr{N}^{-1} \sum_{s=1}^{N} \int d \lambda e^{-\frac{1}{2} \lambda^{2}+y \lambda} H_{N-s}^{2}(\lambda) \prod_{r \neq s} \int d \mu e^{-\frac{1}{2} \mu^{2}} H_{N-r}^{2}(\mu) \\
& =\sum_{s=0}^{N-1} \frac{\int d \lambda e^{-\frac{1}{2} \lambda^{2}+y \lambda} H_{s}^{2}(\lambda)}{\int d \lambda e^{-\frac{1}{2} \lambda^{2}} H_{s}^{2}(\lambda)} .
\end{aligned}
$$

Not only have all the variables $\lambda_{i}$ been decoupled, but the whole calculation reduces now to a single harmonic oscillator

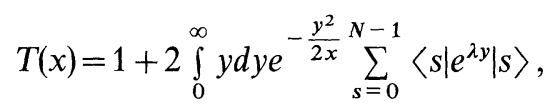

where we have used quantum mechanical notations to denote the orthonormalized wave functions

$$
\langle\lambda \mid s\rangle=\frac{e^{-\frac{\lambda^{2}}{4}} H_{s}(\lambda)}{\left(\int d \lambda e^{-\frac{\lambda^{2}}{2}} H_{s}(\lambda)^{2}\right)^{1 / 2}} .
$$

One introduces the "annihilation" and "creation" operators $a$ and $a^{\dagger}$,

$$
\begin{aligned}
a & =\frac{\lambda}{2}+\frac{\partial}{\partial \lambda}, \\
a^{\dagger} & =\frac{\lambda}{2}-\frac{\partial}{\partial \lambda},
\end{aligned}
$$

which satisfy $\left[a, a^{\dagger}\right]=1, \lambda=a+a^{\dagger}, a|0\rangle=0$ and generate the state $|s\rangle$ according to

$$
|s\rangle=\frac{1}{\sqrt{s !}}\left(a^{\dagger}\right)^{s}|0\rangle .
$$

It is again convenient to introduce generating functions for the quantities $\left\langle s\left|e^{y \lambda}\right| s\right\rangle$,

$$
G(y, v)=\sum_{s} \frac{v^{s}}{s !}\left\langle s\left|e^{y \lambda}\right| s\right\rangle=\sum_{s} \frac{1}{(s !)^{2}}\left\langle 0\left|\left(v^{1 / 2} a\right)^{s} e^{y\left(a+a^{\dagger}\right)}\left(v^{1 / 2} a^{\dagger}\right)^{s}\right| 0\right\rangle
$$

with $v$ positive. If we set $z=v^{1 / 2} e^{i \theta}$, this may be written as

$$
\begin{aligned}
G(y, v) & =\int \frac{d \theta}{2 \pi}\left\langle 0\left|e^{\bar{z} a} e^{y\left(a+a^{\dagger}\right)} e^{z a^{\dagger}}\right| 0\right\rangle \\
& =\int \frac{d \theta}{2 \pi} e^{\frac{1}{2} y^{2}+|z|^{2}+y(z+\tilde{z})} \\
& =\sum_{n=0}^{\infty} \frac{y^{2 n} v^{n}}{(2 n) !} \int \frac{d \theta}{2 \pi}\left(e^{i \theta}+e^{-i \theta}\right)^{2 n} e^{\frac{1}{2} y^{2}+v} \\
& =\sum_{n=0}^{\infty} \frac{y^{2 n} v^{n}}{(n !)^{2}} e^{\frac{1}{2} y^{2}+v}
\end{aligned}
$$


where use has been made of the Baker-Campbell-Hausdorff formula to transform (3.11a) into $(3.11 \mathrm{~b})$. It is now easy to carry out the $y$-integration

$$
\begin{aligned}
\sum_{s} \frac{v^{s}}{s !} \int_{0}^{\infty} d y^{2} e^{-\frac{y^{2}}{2 x}}\left\langle s\left|e^{y \lambda}\right| s\right\rangle & =\int_{0}^{\infty} d y^{2} e^{-\frac{y^{2}}{2 x}} \sum_{n} \frac{y^{2 n} v^{n}}{(n !)^{2}} e^{\frac{1}{2} y^{2}+v} \\
& =\sum_{n=0}^{\infty} \frac{v^{n}}{(n !)^{2}} e^{v}\left(\frac{2 x}{1-x}\right)^{n+1} \Gamma(n+1) \\
& =\frac{2 x}{1-x} e^{v \frac{1+x}{1-x}} \\
& =\frac{2 x}{1-x} \sum_{s=0}^{\infty} \frac{v^{s}}{s !}\left(\frac{1+x}{1-x}\right)^{s},
\end{aligned}
$$

which allows us to identify

$$
\int_{0}^{\infty} d y^{2} e^{-\frac{\lambda^{2}}{2 x}}\left\langle s\left|e^{\lambda y}\right| s\right\rangle=\frac{2 x}{1-x}\left(\frac{1+x}{1-x}\right)^{s},
$$

and thus

$$
T(x)=1+2 \sum_{0}^{\infty} x^{n+1} \frac{\left\langle t_{[2 n]}\right\rangle}{(2 n-1) ! !}=1+\sum_{0}^{N-1} \frac{2 x}{1-x}\left(\frac{1+x}{1-x}\right)^{s}=\left(\frac{1+x}{1-x}\right)^{N},
$$

which is equivalent to the former statement (2.18) and completes this second derivation.

\section{The Virtual Euler Characteristics}

We finally turn to Penner's approach. Using the duality and symmetry noticed above in $(2.10)$, the quantity $\varepsilon_{g}(n)$ of $(2.15)$ may also be computed as

$$
\varepsilon_{g}(n)=\frac{1}{N} \sum_{\substack{\underline{\mu} \\ \Sigma \mu_{i}=n+1-2 g}}\left\langle t_{\underline{\mu}}\right\rangle_{[2 n]} \frac{2 n}{\prod_{k} k^{\mu_{k}} \mu_{k} !} .
$$

The counting implied in $\varepsilon_{g}(n)$ is not quite the one required. In (4.1) we have to discard graphs with vertices of valency one ("tadpoles") or two ("self-energy" insertions), (as in Fig. 2), obtaining the required quantity $\lambda_{g}(n)$. The "virtual" Euler characteristics $\chi\left(\Gamma_{g}^{1}\right)$ of the modular group of once punctured surfaces of genus $g$ is then

$$
\sum_{n=2 g}^{6 g-3}(-1)^{n-1} \frac{\lambda_{g}(n)}{2 n}
$$

where $\lambda_{g}(n)$ is obtained by the same formula (4.1) as $\varepsilon_{g}(n)$ with the additional constraint that $\mu_{1}=\mu_{2}=0$. Thus a superficial view seems to indicate that by computing the coefficient of $N$ in

$$
\Phi(x, N)=\ln Z(x, N)=\ln \frac{\int d M e^{-\frac{1}{x^{2}} \operatorname{tr} \sum_{2}^{\infty} \frac{M^{k} x^{k}}{k}}}{\int d M e^{-\frac{1}{2} \operatorname{tr} M^{2}}}
$$


one obtains the asymptotic expansion $\sum_{g} x^{4 g-2} \chi\left(\Gamma_{g}^{1}\right)$. This is, however, dangerous since as it stands the numerator of (4.3) is not defined. The situation is analogous (and the analogy is of course not fortuitous) to the one prevailing in the derivation of Stirling's formula for Euler's $\Gamma$ function. Starting from

$$
\Gamma(s+1)=\int_{0}^{\infty} d u e^{-u+s \ln u}
$$

for $s$ positive and setting $u=s-v \sqrt{s}$,

$$
s \Gamma(s)=\left(\frac{s}{e}\right)^{s} \sqrt{s} \int_{-\infty}^{\sqrt{s}} d v e^{-s \sum_{2}^{\infty} \frac{1}{k}\left(\frac{v}{\sqrt{s}}\right)^{k}}
$$

we find for $x$ real and positive

$$
\frac{\left(e x^{2}\right)^{\frac{1}{x^{2}}}}{\sqrt{2 \pi x^{2}}} \Gamma\left(\frac{1}{x^{2}}\right)=\frac{\int_{-\infty}^{\frac{1}{x}} d m e^{-\frac{1}{x^{2}}{ }_{k \geqq 2} \frac{m^{k} x^{k}}{k}}}{\int_{-\infty}^{\infty} d m e^{-\frac{1}{2} m^{2}}} .
$$

The above integral is well defined and sensible. In an asymptotic expansion for $x \rightarrow+0$, we can, however (up to exponentially small turns), take the upper limit to $+\infty$ and obtain term by term the coefficients as sums over simple graphs. This is the sense in which one can understand Eq. (4.3) provided (i) one assumes $x \rightarrow+0$; (ii) one reduces it to an integral over the eigenvalues $\lambda_{i}$ of $M$, each one restricted to $\lambda_{i}<1 / x$. The computation is straightforward

$$
Z(x, N)=\frac{1}{(2 \pi)^{N / 2} \prod_{1}^{N} p !} \int \ldots \int \prod_{1}^{N}\left(d \lambda_{i} \theta\left(1-\lambda_{i} x\right)\left(1-\lambda_{i} x\right)^{\frac{1}{x^{2}}} e^{\frac{\lambda_{i}}{x}}\right) \Delta^{2}(\lambda),
$$

where $\theta$ is the step function. Changing variables to $y_{i}=\left(1-x \lambda_{i}\right) / x^{2}$ we readily obtain

$$
Z(x, N)=\left[\frac{\left(e x^{2}\right)^{\frac{1}{x^{2}}}}{\sqrt{2 \pi}}\right]^{N} \frac{x^{N^{2}}}{\prod_{1}^{N-1} p !} \operatorname{det}\left(\Gamma\left(\frac{1}{x^{2}}+r+s+1\right)\right)_{0 \leqq r, s \leqq N-1} .
$$

Using

$$
\Gamma\left(1+\frac{1}{x^{2}}+n\right)=\left(\frac{1}{x^{2}}\right)^{n+1} \prod_{1}^{n}\left(1+p x^{2}\right) \Gamma\left(\frac{1}{x^{2}}\right)
$$

we have

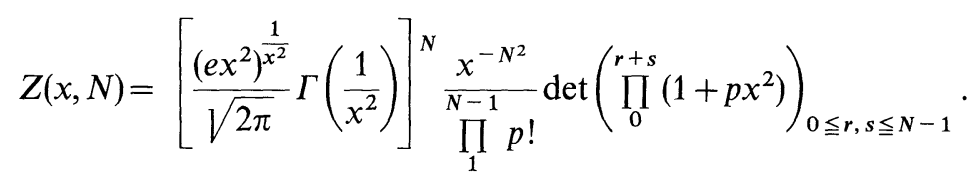



The last determinant is evaluated as $x^{N(N-1)} \prod_{1}^{N-1} p ! \prod_{1}^{N-1}\left(1+p x^{2}\right)^{N-p}$ so that we
obtain

$$
Z(x, N)=\left[\frac{\left(e x^{2}\right)^{\frac{1}{x^{2}}}}{\sqrt{2 \pi x^{2}}} \Gamma\left(\frac{1}{x^{2}}\right)\right]_{1}^{N} \prod_{1}^{N-1}\left(1+p x^{2}\right)^{N-p} .
$$

To extract the required quantities we now use Stirling's formula $(x \rightarrow+0)$,

$$
\ln \left[\frac{\left(e x^{2}\right)^{\frac{1}{x^{2}}}}{\sqrt{2 \pi x^{2}}} \Gamma\left(\frac{1}{x^{2}}\right)\right] \simeq \sum_{n=1}^{\infty} \frac{B_{2 n}}{2 n(2 n-1)} x^{4 n-2}
$$

where the symbol $\simeq$ means the equality of (right) derivatives as $x \rightarrow 0$ (of course the right-hand side diverges, more properly it should be written $\left.\sum_{n=1}^{k-1} \frac{B_{2 n}}{2 n(2 n-1)} x^{4 n-2}+O\left(x^{4 k-2}\right)\right)$. We also introduce the Bernoulli polynomials

$$
\begin{gathered}
\frac{t e^{t x}}{e^{t}-1}=\sum_{n=0}^{\infty} B_{n}(x) \frac{t^{n}}{n !}, \\
B_{n}(x)=\sum_{r=0}^{n}\left(\begin{array}{l}
n \\
r
\end{array}\right) B_{r} x^{n-r},
\end{gathered}
$$

such that for $k \geqq 1$,

$$
\sum_{p=1}^{N-1} p^{k}=\frac{B_{k+1}(N)-B_{k+1}}{k+1}
$$

Therefore, when $x \rightarrow+0$

$$
\begin{aligned}
\Phi(x, N)= & \ln Z(x, N) \\
\simeq & N \sum_{n=1}^{\infty} \frac{B_{2 n}}{2 n(2 n-1)} x^{4 n-2} \\
& +\sum_{k=1}^{\infty}(-1)^{k-1} \frac{x^{2 k}}{k}\left[\frac{N}{k+1} \sum_{r=1}^{k+1} N^{r}\left(\begin{array}{c}
k+1 \\
r
\end{array}\right) B_{k+1-r}\right. \\
& \left.-\frac{1}{k+2} \sum_{r=1}^{k+2} N^{r}\left(\begin{array}{c}
k+2 \\
r
\end{array}\right) B_{k+2-r}\right] .
\end{aligned}
$$

The coefficient of $N$ is the asymptotic series

$$
\sum_{g=1}^{\infty} \frac{B_{2 g}}{2 g(2 g-1)} x^{4 g-2}+\sum_{k=1}^{\infty}(-1)^{k} \frac{x^{2 k}}{k} B_{k+1}
$$

For $k \geqq 1, B_{k+1}$ vanishes unless $k+1$ is even, therefore,

$$
\sum \chi\left(\Gamma_{g}^{1}\right) x^{4 g-2} \simeq-\sum_{g=1}^{\infty} x^{4 g-2} \frac{B_{2 g}}{2 g}
$$


or alternatively (for $g>0$ )

$$
\chi\left(\Gamma_{g}^{1}\right)=-\frac{B_{2 g}}{2 g}=\zeta(1-2 g) .
$$

Interestingly, the general coefficient of $N^{s}$ in $\Phi$ (connected diagrams with $s$ faces) is (for $g>0$ )

$$
\sum_{2 g>\sup (0,2-s)} x^{4 g-4+2 s}(-1)^{s} \frac{B_{2 g}}{2 g(2 g+s-2)}\left(\begin{array}{c}
2 g+s-2 \\
s
\end{array}\right) .
$$

According to Penner, the coefficient of this expansion is the virtual Euler characteristics $\chi\left(\Gamma_{g}^{s}\right)$ of the mapping class group of a Riemann surface of genus $g$ with $s$ punctures (allowed to be permuted) so that for $s>0,2 g-2+s>0$, one has

$$
\chi\left(\Gamma_{g}^{s}\right)=(-1)^{s-1} \frac{\zeta(1-2 g)}{2 g+s-2}\left(\begin{array}{c}
2 g+s-2 \\
s
\end{array}\right) .
$$

In conclusion it is perhaps worth mentioning that by evaluating integrals over matrices one can expect to capture more information on various aspects of spaces of Riemann surfaces and find a bridge with current developments in conformal field theory.

\section{References}

1. Harer, J., Zagier, D.: Invent. Math. 85, 457-485 (1986)

2. Penner, R.C.: Bull. Am. Math. Soc. 15, 73-77 (1986); J. Diff. Geom. 27, 35-53 (1988)

3. 't Hooft, G.: Nucl. Phys. B 72, 461-473 (1974)

4. Brézin, E., Itzykson, C., Parisi, G., Zuber, J.-B.: Comm. Math. Phys. 59, 35-51 (1978)

5. Bessis, D.: Commun. Math. Phys. 69, 147-163 (1979)

6. Bessis, D., Itzykson, C., Zuber, J.-B.: Adv. Appl. Math. 1, 109-157 (1980)

7. Itzykson, C., Zuber, J.-B.: J. Math. Phys. 21, 411-421 (1980)

8. Ivanov, N.V.: Usp. Mat. Nauk 42, 49-91 (1987); Russian Math. Surv. 42, 55-107 (1987)

9. Weyl, H.: The classical groups. Princeton, NJ: Princeton University Press 1946

Communicated by K. Gawedzki 
